\title{
光质对豇豆幼苗环旋运动的影响
}

\author{
李 奇 胡 飞* \\ 华南农业大学农学院, 广州 510642
}

摘 要 为研究光质对哣豆(Vigna unguiculata)幼苗环旋运动的影响, 分别在暗箱的顶部和侧面固定一台红外检测仪, 在垂 直生长方向分别设置白、红、蓝、红-蓝光源, 对豇豆幼苗生长点运动进行 $48 \mathrm{~h}$ 的等间隔 $(5 \mathrm{~min})$ 拍照, 读取图片中豇豆幼苗生 长点的轨迹坐标进行分析。结果表明: 黑暗下, 豇豆幼苗生长点的运动分为逆时针螺旋上升(约占 $1 / 3$ )和不规则的摆动上升(约 占 $2 / 3$ )两类。螺旋上升持续时间为 $18.3 \mathrm{~h}$, 后转为不规则的摆动上升, 环旋一周的时间为 $(82.7 \pm 4.2) \mathrm{min}$, 最大摆幅为 $2.0 \mathrm{~cm}$, 最小摆幅为 $0.5 \mathrm{~cm}$ 。白光下为趋向光源摆动上升, 摆动幅度由大到小, 再变大, 最大摆幅为 $0.6 \mathrm{~cm}$, 最小摆幅为 $0.1 \mathrm{~cm}$ 。红光 下为近直线趋向光源和垂直光源方向摆动两个阶段, 近直线趋向光源持续(21.9 \pm 1.6$) h$, 垂直光源方向摆动持续 $(8.8 \pm 0.5) h$, 最大摆幅为 $3.5 \mathrm{~cm}$, 最小摆幅为 $2.0 \mathrm{~cm}$ 。蓝光下为“Z”形向光源上升运动, (110.0 \pm 5.8$) \min$ 和 $(223.5 \pm 4.9) \min$ 两种周期交替进 行。红-蓝光下趋向蓝光运动持续(12.0 \pm 3.8$) \mathrm{h}$ 后, 转慢速趋红光运动。不同光质下 $48 \mathrm{~h}$ 内豇豆幼苗增长高度由高到低为: 红 光 $>$ 黑暗 $>$ 蓝光 $>$ 白光 $>$ 红-蓝光。豇豆幼苗在没有光照下也产生环旋运动, 环旋运动不需要光的诱导, 但光质改变了幼苗运动 的方向和速度。不同光质下豇豆幼苗有不同的运动形式, 但总体上都表现为趋光性。

关键词 环旋运动; 光质; 三维坐标; 豇豆

李奇, 胡飞 (2018). 光质对豇豆幼苗环旋运动的影响. 植物生态学报, 42, 1192-1199. DOI: 10.17521/cjpe.2018.0038

\section{Effects of light quality on circumnutation of Vigna unguiculata seedlings}

LI Qi and HU Fei

College of Agriculture, South China Agricultural University, Guangzhou 510642, China

\section{Abstract}

Aims The aim of this study is to investigate the effect of different light qualities on circumnutation of cowpea (Vigna unguiculata) seedlings.

Methods The behaviors of cowpea seedlings were observed in a black box, which fixed with two infrared cameras on the top and a side, respectively. The motion was recorded as photos taken at an interval of 5 min, in a 3-dimensional space coordinate under different light qualities (such as white, red, blue, and red-blue with opposite direction) for $48 \mathrm{~h}$. The coordinate values of cowpea seedling growth point (CPGP) were read on a computer screen.

Important findings The results showed that in the dark condition, the CPGP movements had two types, one was a spiral rising anticlockwise circulation, and the other was swinging up irregularly. The spiral rising anticlockwise circulation lasted $18.3 \mathrm{~h}$, then turned to swing up irregularly. It takes $(82.7 \pm 4.2)$ min spiraling a circle on average, with the maximum range of swing $2.0 \mathrm{~cm}$ and the minimum $0.5 \mathrm{~cm}$. Under white light condition, the CPGP movements tended toward light source with a swing up irregularly. The range of swing changed from decrease to increase, with the maximum range of swing $0.6 \mathrm{~cm}$ and the minimum $0.1 \mathrm{~cm}$. Under red light condition, the CPGP movements showed two stages, in which one was a tending toward light source directly for $(21.9 \pm 1.6) \mathrm{h}$, the other was swing up perpendicular to light direction for $(8.8 \pm 0.5) \mathrm{h}$. The maximum range of swing was $3.5 \mathrm{~cm}$, and the minimum was $2.0 \mathrm{~cm}$. Under blue light condition, the CPGP movements showed a swing up with " $Z$ " trajectory tending toward light source, alternating 2 durations with $(110.0 \pm 5.8) \mathrm{min}$ and $(223.5 \pm 4.9)$ min. under red-blue light with opposite direction conditions, the CPGP movements tended toward blue light source for $(12.0 \pm$ 3.8) h, then toward red light source slowly. The order of CPGP within $48 \mathrm{~h}$ decreased under different light quality with red, dark, blue, white and red-blue. The seedlings of cowpea could move with circumnutation in dark condi-

收稿日期Received: 2018-02-06 接受日期Accepted: 2018-09-26

基金项目：国家自然科学基金(30470192)。Supported by the National Natural Science Foundation of China (30470192).

* 通信作者Corresponding author (hufei@scau.edu.cn) 
tions, suggesting that circumnutation does not need light induction. However, the light quality could change the movement direction and speed. CPGP movements showed different patterns under different light qualities, but the movements tended toward light source in general.

Key words circumnutation; light quality; 3-dimensional space coordinate; Vigna unguiculata

Li Q, Hu F (2018). Effects of light quality on circumnutation of Vigna unguiculata seedlings. Chinese Journal of Plant Ecology, 42, 1192-1199. DOI: $10.17521 /$ cjpe.2018.0038

环旋运动在植物界中普遍存在(Darwin, 1865, 1876; Darwin \& Darwin, 1880), 它是植物的某些器 官如根、芽等在生长过程中所做出的一种生长运动 (Brown, 1993), 大致可描述为钟摆状、椭圆形或圆 形等(Melin, 1975; 刘公社和张俊英, 1994)。影响植 物环旋运动的因素很多, 有研究发现其受重力的调 节(Brown \& Chapman, 1977; Yoshihara \& Iino, 2006; Kim et al., 2016)。Mccomb (1962)认为赤霉素处理对 踠豆(Pisum sativum) 植株的环旋运动有明显的促进 作用。而光作为植物生长和形态建成的条件, 对植 物不同器官的生长运动方向有重要的影响, Iida等 (2018)发现赤豆(Vigna angularis)的上胚轴在白光下 顺时针环旋运动, 其运动周期为 $60 \mathrm{~min}$ 。Stolarz和 Dziubińska (2017)通过改变光暗条件发现不同光强 对向日葵(Helianthus annuus)幼苗的环旋运动具有 调节作用, 弱光下运动周期较长。也有人发现蓝光 能够稍微提高踠豆卷须环旋运动的速率, 而红外线 照射(即热量梯度)影响其方向的改变(Jaffe, 1970)。

前人多在二维坐标里对环旋运动进行观测和描 述, 事实上植物环旋运动过程也伴随着生长运动。 二维坐标里只能反映这一复杂运动的某一个侧面, 显然有一定的局限性。本研究参考Gill等(2009)的经 验, 自制了一个三维空间坐标装置, 其优点在于不 影响植物自然生长, 可以连续地测量不同光质下豇 豆(Vigna unguiculata)幼苗在时空上的生长运动轨 迹。利用该装置对豇豆幼苗运动方式、运动幅度和 周期进行了测定, 以探讨不同光质对豇豆幼苗环旋 运动的影响, 为探索植物环旋运动的影响条件与机 理提供参考。

\section{1 材料和方法}

\section{1 供试材料}

供试材料为豇豆, 品种为“油白”。在装有湿润水 稻土(取自华南农业大学农场)的塑料盒(直径 $15.2 \mathrm{~cm}$, 高8.6 cm)中心播种。每盒播种1穴, 每穴2粒, 播种 深度2.0-3.0 cm。用A4纸覆盖塑料盒, 播种穴处留直
径 $1 \mathrm{~cm}$ 的孔, 在培养箱(RTOP-500D, 浙江托普仪器 有限公司, 杭州)中 $(30 \pm 1){ }^{\circ} \mathrm{C}$ 黑暗条件培养 3 天, 豇豆幼苗出土2.00-3.00 cm时, 保留其中 1 株用于试 验。

\section{2 空间坐标的制作}

参考 $3 \mathrm{D}$ 观测根生长运动的设计 (Gill et al., $2009)$, 把两块 $40.0 \mathrm{~cm} \times 30.0 \mathrm{~cm}$ 的方形泡沫板黏合 在一起, 呈 “L”形(图 1)。在一张长 $70.0 \mathrm{~cm}$, 宽 $35.0 \mathrm{~cm}$ 的白色卡片纸上用 $0.5 \mathrm{~mm}$ 油性笔画出边长 为 $1.0 \mathrm{~cm}$ 的网格, 然后固定在“ $\mathrm{L}$ ”形的泡沫板上, 并 标出 $x$ 轴与 $y$ 轴。其中, $x$ 轴在水平泡沫板上, $y$ 轴从水 平板的中间经过“ “ $"$ ”形的拐角一直延伸到坚直板的 上端, 坚直方向为 $z$ 轴, 如图 1 所示。然后在水平板上 的坐标原点处挖去一个边长为 $1.0 \mathrm{~cm}$ 的正方形小孔, 原点为小孔的中心。并沿着小孔与 $y$ 轴平行的一边, 把泡沫板连同制定的坐标纸一起切开, 同时沿着小 孔与 $y$ 轴平行的另一边只把泡沫板切开, 这样就形 成了一个 $1.0 \mathrm{~cm}$ 的通路, 方便试验材料的换取, 同 时, 把切下来的泡沫板长条黏合在坐标纸上, 当把 试验材料放进坐标原点时, 就可以把通路封住, 同 时保证了坐标纸的平展性(图1)。

\section{3 不同光质光源制作}

采用USB可调节方向的LED灯( (6000 \pm 200$)$ Lx) 2 个。透红色与蓝色光聚乙烯膜各一张, 然后用 剪刀剪成适合尺寸, 紧贴于LED灯上, 制成红、蓝光 源(红光波长: $660 \mathrm{~nm}$, 蓝光波长: $450 \mathrm{~nm}$ ), 白光源 不贴有色膜。
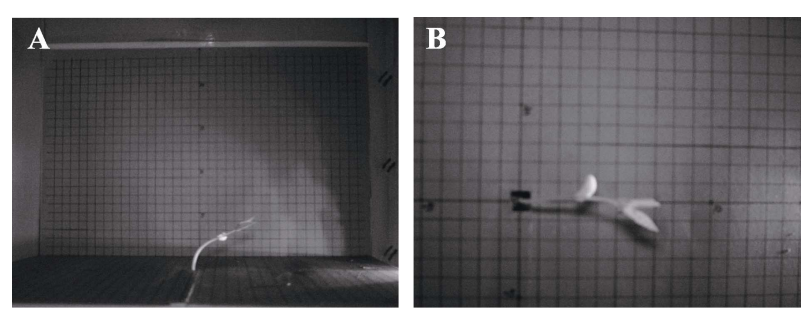

图1 豇豆幼苗环旋运动的空间坐标示意图。 $\mathrm{A}$, 侧视图。 $\mathrm{B}$, 俯视图。

Fig. 1 Space coordinates diagram of circumnutation of Vigna unguiculata seedlings. A, Side view. B, Top view. 


\section{4 数据的获取}

把制作好的空间坐标放入长 $72.0 \mathrm{~cm}$, 宽 $42.0 \mathrm{~cm}$, 高 $47.0 \mathrm{~cm}$ 的不透光的硬纸箱中, 并在其 “L”空间坐标的坚直板对面固定一台红外检测仪 (SG-008, 深圳市思远数码技术有限公司), 使其拍 照的高度正好与放入试验材料后的空间坐标水平板 平行检测仪(a), 在纸箱顶部即空间坐标水平板的上 方固定另一台红外检测仪(b), 使其拍照范围正好以 坐标原点为中心(图1)。在 $x$ 轴正方向上, 在与空间坐 标水平板平行的高度开出一个边长为 $2.0 \mathrm{~cm}$ 的正方 形小孔, $x$ 轴正好从小孔的中心穿过。把不同光质的 光源固定在小孔上, 持续稳定发光, 两台检测仪同 时运行, 检测间隔为 $5 \mathrm{~min}$, 分辨率为 1200 万像素, 连续检测 $48 \mathrm{~h}$ 。人工读取豇豆生长点在照片上的坐 标。每次 1 株, 3 个重复。

\section{2 结果和分析}

\section{1 黑暗下豇豆幼苗生长点的运动规律}

黑暗情况下在观测的 $48 \mathrm{~h}$ 内, 1 株豇豆幼苗生长 点分为两个阶段, 先逆时针螺旋上升运动, 再进行
无规律摆动上升运动(图2A), 2株为无规律摆动上 升。图2A中的起始坐标为 $(0.4,-0.8,2.0$, 试验中把 豇豆幼苗贴近泥土部位的胚轴放在空间坐标原点上, 但豇豆幼苗的生长点与其贴近泥土部分的胚轴并不 是完全垂直, 因此豇豆幼苗的起始位点不在原点, 下同)。生长点运动分为两阶段的植株逆时针螺旋运 动上升一周的时间为 $(82.7 \pm 4.2) \mathrm{min}$, 在二维平面 上，这种运动可描述为逆时针近椭圆形运动(图2B, $x-y$ 平面), 图2B中 $x-z$ 和 $y-z$ 平面反映了该运动的振幅 变化由小变大再变小, 其中最小短轴为 $0.5 \mathrm{~cm}$, 最 大长轴为 $2.0 \mathrm{~cm}$, 该运动持续了约 $18.3 \mathrm{~h}$, 此后为无 规律摆动上升运动(图2A)。

无规律的摆动上升运动阶段在二维平面上可描 述为不规则运动(图2C, $x-y$ 平面), 该运动的方向、摆 动时间变化没有明显的规律。无规律摆动上升运动 的2株与做螺旋上升运动的1株豇豆幼苗生长量相近, 在 $48 \mathrm{~h}$ 内分别生长了 $(13.0 \pm 1.6) \mathrm{cm}$ 和 $12.4 \mathrm{~cm}$ 。

\section{2 白光下豇豆幼苗生长点运动规律}

白光 $48 \mathrm{~h}$ 内 3 株豇豆幼苗生长点运动方式相似, 均为摆动上升运动。图 $3 \mathrm{~A}$ 是随机选取其中一株的三
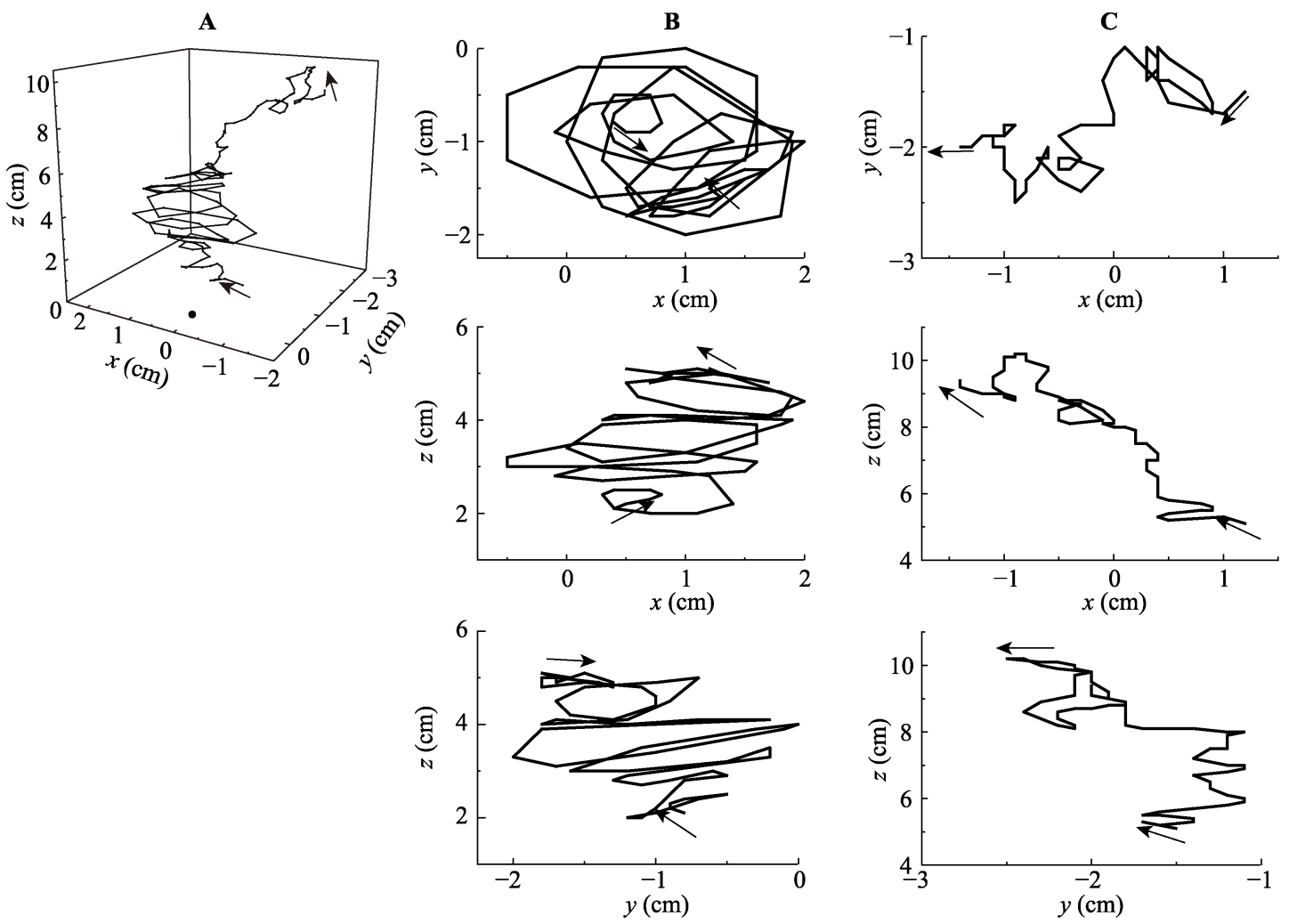

图2 黑暗情况下豇豆幼苗48 h运动轨迹。 $\mathbf{A}$, 三维空间运动轨迹。 $\mathbf{B}$, 椭圆形运动。 $\mathbf{C}$, 不规则运动。, , 坐标原点位置。实线 箭头表示运动方向。

Fig. 2 The trajectory of cowpea seedling in the darkness for 48 h. A, Three-dimensional trajectory. B, Elliptical type. C, Irregular type. $\cdot$, origin position of coordinates. Solid arrows indicate the direction of the movement.

www.plant-ecology.com 
维轨迹图, 起始坐标为 $(1.0,0.8,2.7)$, 由图3B可以 看出, 该运动在二维平面上可描述为钟摆型运动, 该运动在 $x-y$ 和 $y-z$ 平面图上平行于 $y$ 轴方向摆动, $x-z$ 平面投影可以看出这种运动趋向光源, $y-z$ 平面图反 映了该钟摆型运动的摆幅变化为先由大变小, 后又 增大, 其中最小摆幅为 $0.1 \mathrm{~cm}$, 最大摆幅为 $0.6 \mathrm{~cm}$ 。 试验中 3 株豇豆幼苗 $48 \mathrm{~h}$ 内生长了 $(5.4 \pm 0.29) \mathrm{cm}$ 。

\section{3 红光下豇豆幼苗生长点运动规律}

红光下 $48 \mathrm{~h}$ 内 3 株豇豆幼苗生长点运动方式相 似, 先是近直线趋向光源上升, 然后垂直光源方向 上升。随机选取其中 1 株的三维轨迹(图4A), 起始坐 标为 $(-0.2,-0.2,2.2)$ 。 $48 \mathrm{~h}$ 内, 在 $x-y$ 二维平面上可描 述为先近直线型 (图4B), 接着为垂直光源方向的摆 动型(图4C)。近直线型运动在趋向光源的阶段生长 点高度先降低, 后又逐渐升高(图4B, $x-z$ 和 $y-z$ 平面), 该阶段持续了 $(21.9 \pm 1.63) \mathrm{h}$, 随后转变为垂直光 源方向来回摆动, 摆动的时间为 $(8.8 \pm 0.51) \mathrm{h}$, 摆 幅最大为 $3.5 \mathrm{~cm}$, 最小为 $2.0 \mathrm{~cm}$ (图 $4 \mathrm{C}, x-\mathrm{z}$ 和 $y-z$ 平 面)。豇豆幼苗 $48 \mathrm{~h}$ 内生长了 $(13.6 \pm 0.19) \mathrm{cm}$ 。

\section{4 蓝光下豇豆幼苗生长点的运动规律}

蓝光下 $48 \mathrm{~h}$ 内 3 株豇豆幼苗生长点运动方式均 为折线向光源上升, 为“ $\mathrm{Z}$ ”形周期运动。图 $5 \mathrm{~A}$ 是随机 选取其中一株的三维轨迹图, 起始坐标为 $(-0.8$, $-0.4,3.0)$ 。在 $x-y, x-z, y-z$ 平面上均表现为“ $Z$ ”形轨迹,
单个 “ $\mathrm{Z}$ ”形运动如图 5B 所示。豇豆幼苗生长 点完成一个“Z”形运动的时间有两种, 分别为(110.0 $\pm 5.77) \mathrm{min}$ 和(223.5 \pm 4.91$) \mathrm{min}$, 且这两种运动周期 交替发生。 $48 \mathrm{~h}$ 豇豆幼苗生长了 $(10.9 \pm 0.46 \mathrm{~cm})$ 。

\section{5 红-蓝光下豇豆幼苗生长点的运动规律}

红-蓝光下3株豇豆幼苗生长点的运动均是先趋 向蓝光, 后转为趋向红光运动。随机选取其中一株 的三维轨迹(图6A), 豇豆幼苗的起始坐标为 $(-1,0$, 3.0)。在二维平面上, 图6B所示为豇豆幼苗趋向于蓝 光的运动, 该运动持续 $(12.0 \pm 3.75) \mathrm{h}$ 后转为持续慢 速趋向红光方向运动(图6C), $48 \mathrm{~h}$ 豇豆幼苗生长点 偏向红光方向仅 $1.0 \mathrm{~cm}$ 。 $48 \mathrm{~h}$ 内豇豆幼苗生长了(3.2 $\pm 0.8) \mathrm{cm}$ 。

\section{3 讨论和结论}

植物存在着多种运动形式, 这些运动形式通常 与植物种子传播、花粉传输、自身防御和营养获取 有关。如何描述植物运动, 理解植物运动的效应一 直是植物学领域有趣而又没有在机理上全面合理解 释的课题(Skotheim \& Mahadevan, 2005)。100多年来, 植物的环旋运动一直受到植物学家的关注, 以植物 的根、茎、叶、肧轴等器官为研究对象, 分别从重 力(Solheim et al., 2006; Johnsson et al., 2009; Johnsson \& Israelsson, 2010; Scherer \& Pietrzyk, 2014)、激

A
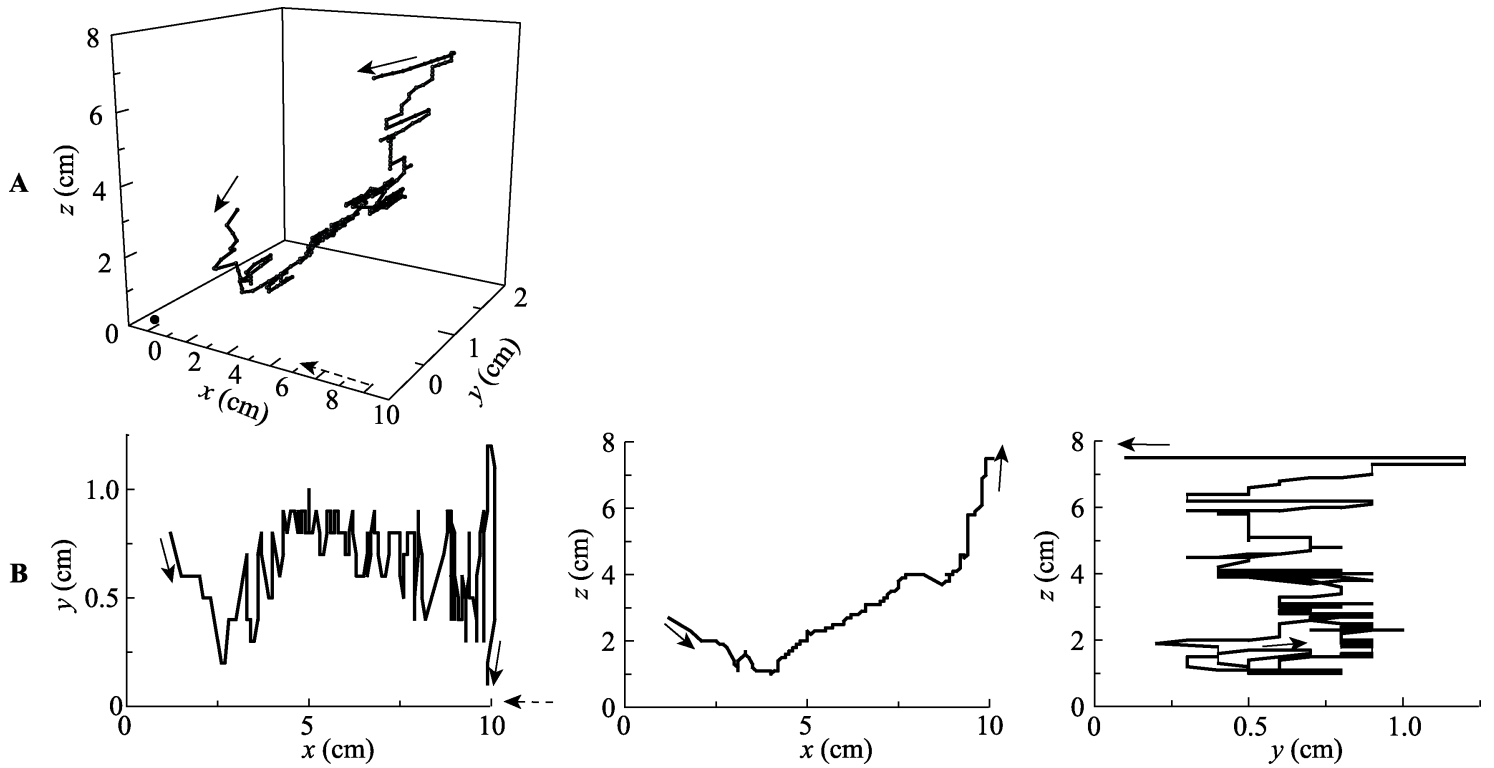

图3 白光下豇豆幼苗48 $\mathrm{h}$ 运动轨迹。 $\mathrm{A}$, 三维空间运动轨迹。 $\mathbf{B}$, 摆动运动。 $\cdot$, 坐标原点位置。实线箭头表示运动方向。光 源中心通过坐标原点, 虚线箭头表示光源方向。

Fig. 3 The trajectory of cowpea seedling in the white light for 48 h. A, Three-dimensional trajectory. B, Perpendicular type. •, origin position of coordinates. Solid arrows indicate the direction of the movement. Light source center went through the origin of coordinates and short dash arrows indicate the light source direction. 

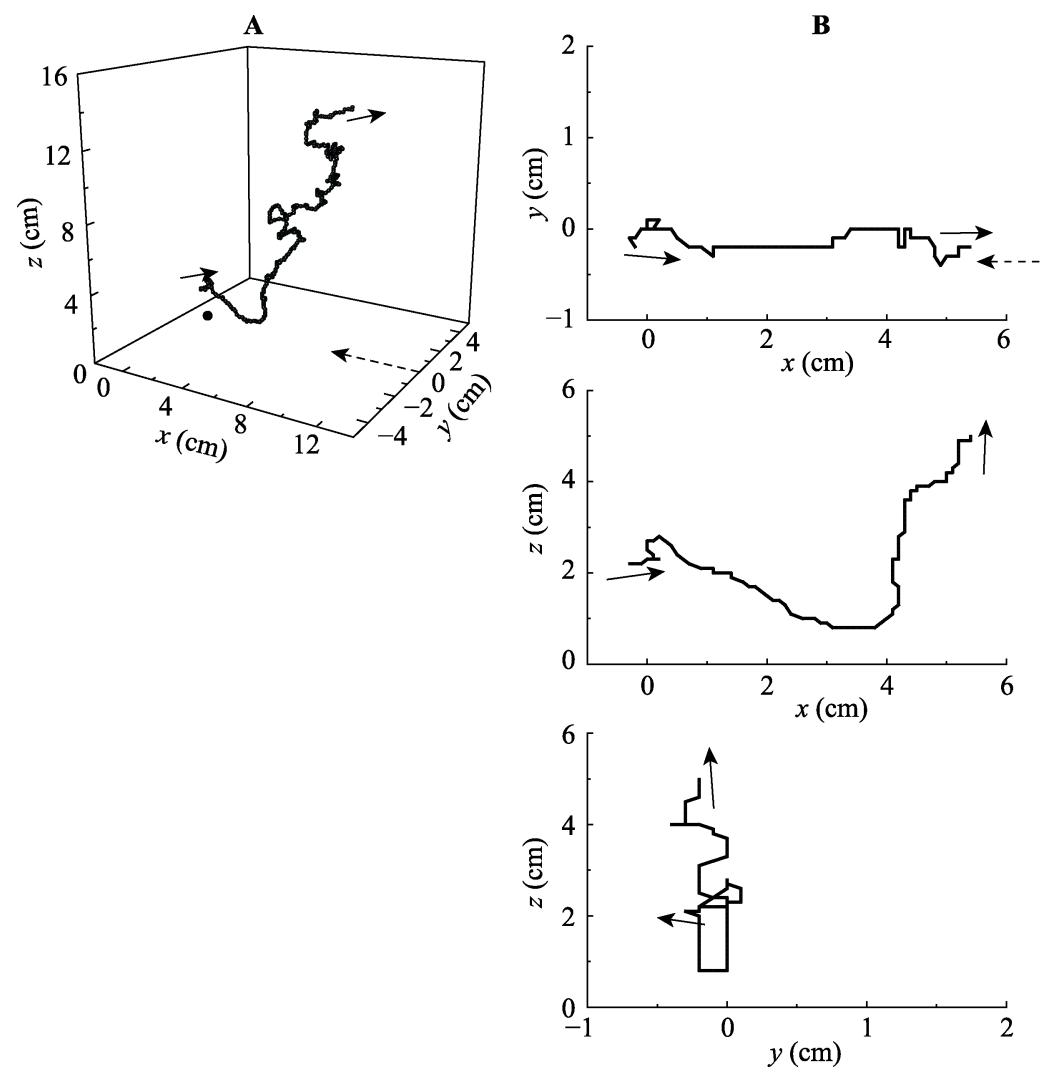
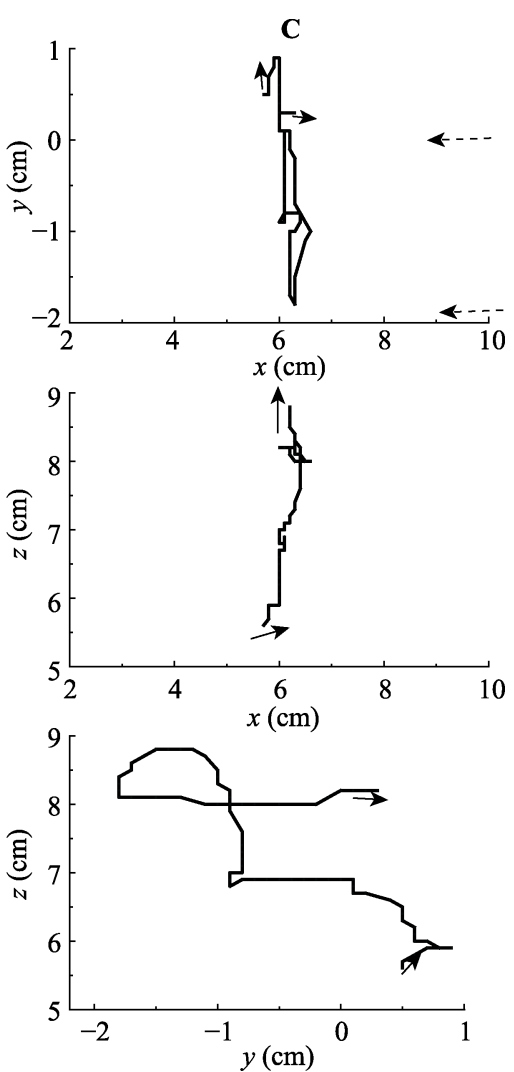

图4 红光下豇豆幼苗48 h运动轨迹。 $\mathbf{A}$, 三维空间运动轨迹。 $\mathbf{B}$, 近直线运动。 $\mathrm{C}$, 垂直光源方向摆动型运动。 $\cdot$, 坐标原点位 置。光源中心通过坐标原点，虚线箭头表示光源方向。实线箭头表示运动方向。

Fig. 4 The trajectory of cowpea seedling in the red light for $48 \mathrm{~h}$. A, Three-dimensional trajectory. B, Near linear type. C, Swing up perpendicular to light direction type. •, origin position of coordinates. Light source center went through the origin of coordinates and short dash arrows indicate the light source direction. Solid arrows indicate the direction of the movement.
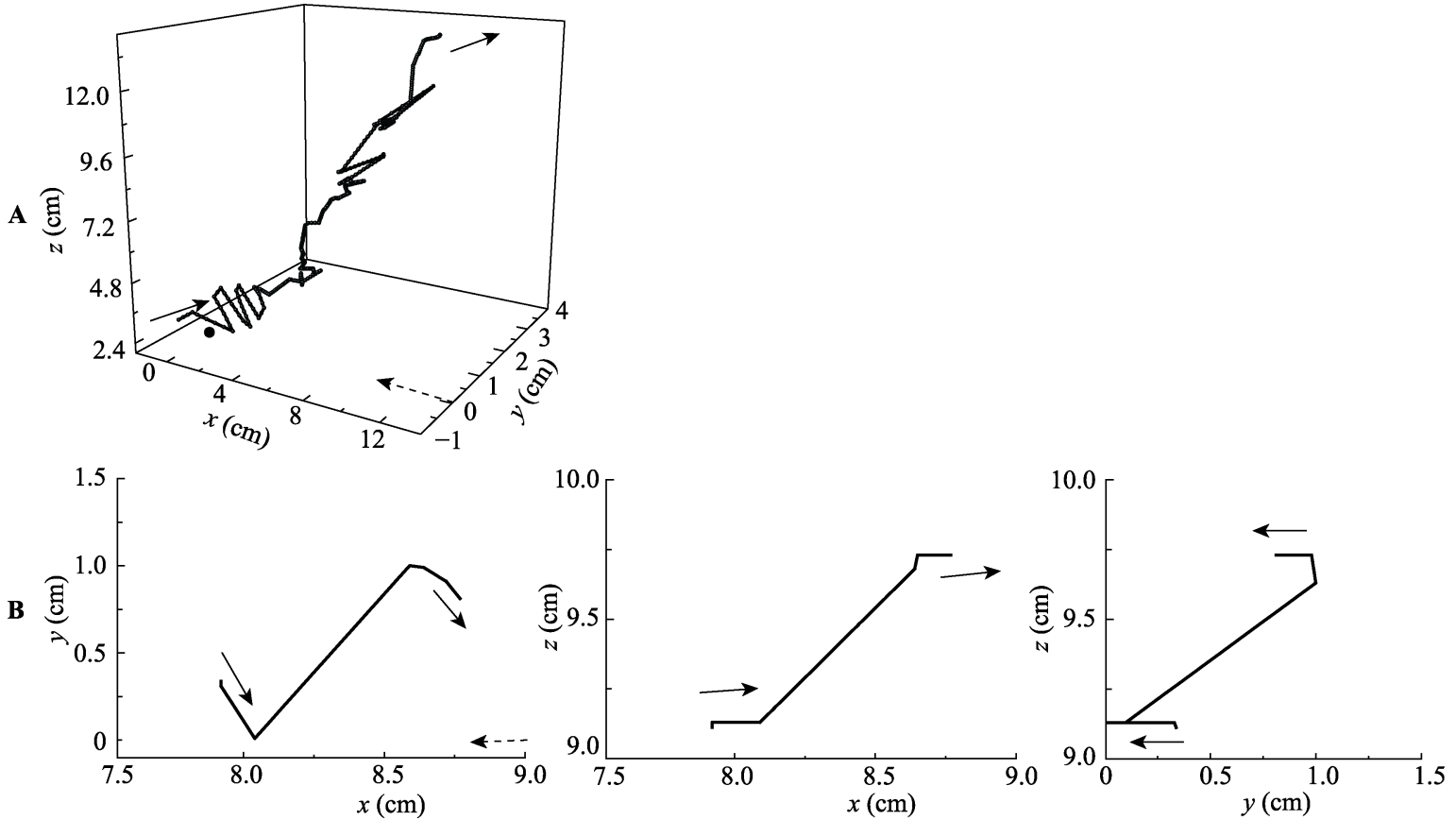

图5 蓝光下豇豆幼苗48 h运动轨迹。 $\mathbf{A}$, 三维空间运动轨迹。 $\mathbf{B}, “ \mathrm{Z}$ ”形运动。, , 表示坐标原点位置。光源中心通过坐标原点, 虚线箭头表示光源方向。实线箭头表示运动方向。

Fig. 5 The trajectory of cowpea seedling in the blue light for $48 \mathrm{~h}$. A, Three-dimensional trajectory. B, "Z” shape. •, origin position of coordinates. Light source center went through the origin of coordinates and short dash arrows indicate the light source direction. 
Solid arrows indicate the direction of the movement.

$\mathbf{A}$

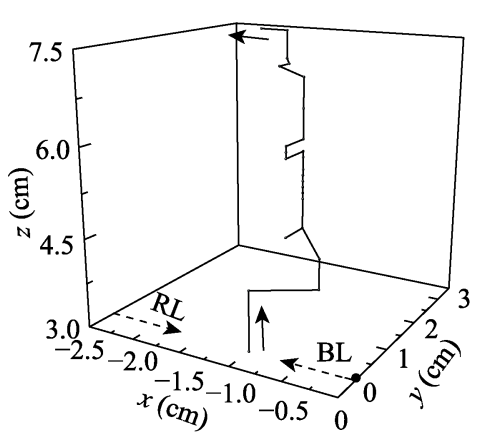

B
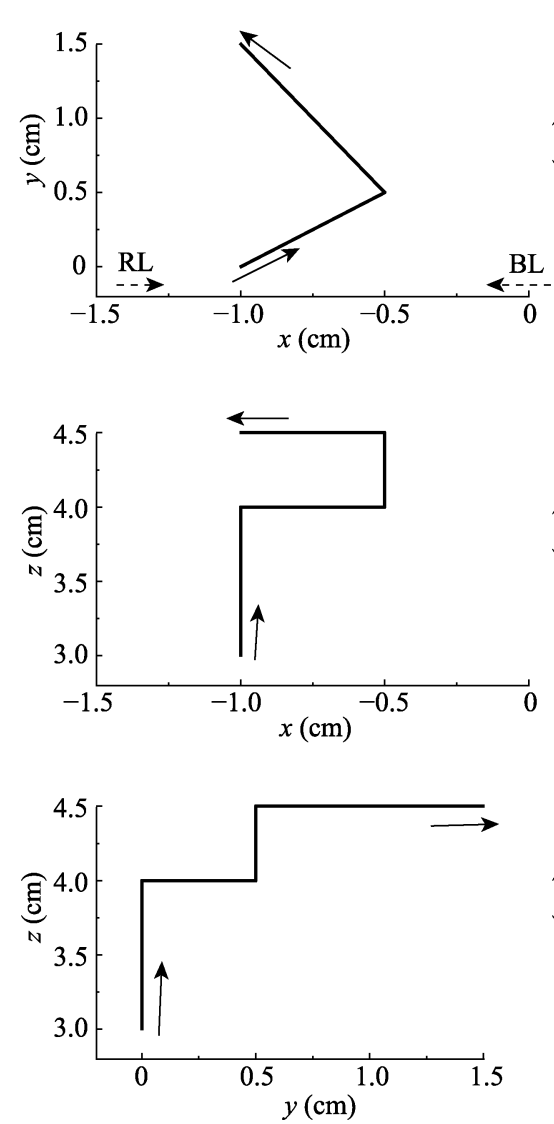
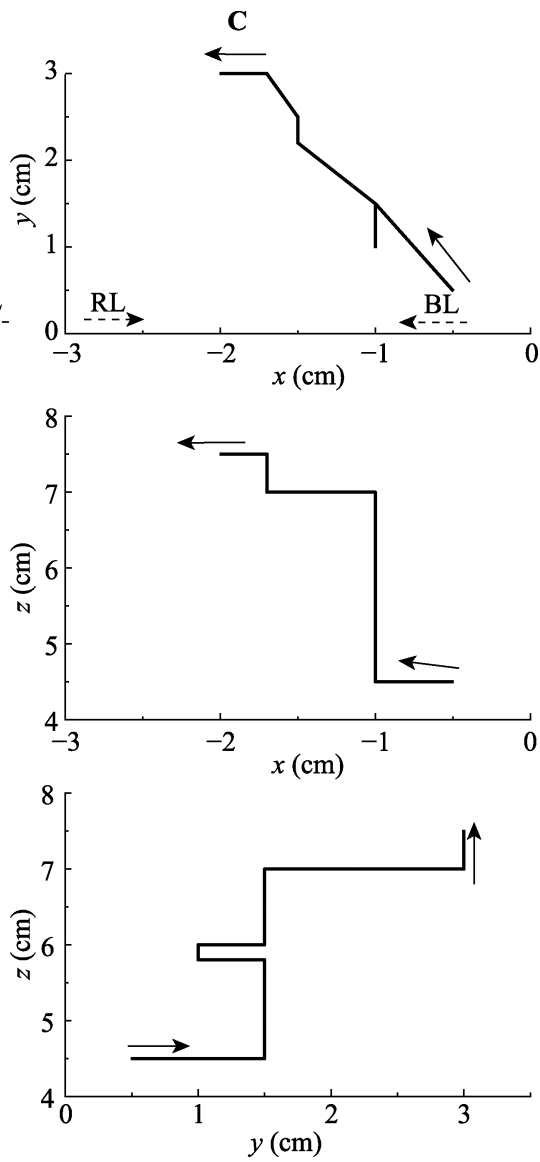

图6 红-蓝光下豇豆幼苗48 h运动轨迹。 $\mathbf{A}$, 三维空间运动轨迹。 $\mathbf{B}$, 趋向蓝光运动。 $\mathbf{C}$, 趋向红光运动。 $\mathrm{BL}$, 蓝光; RL, 红光。 •, 坐标原点位置。光源中心通过坐标原点, 虚线箭头表示光源方向。实线箭头表示运动方向。

Fig. 6 The trajectory of cowpea seedling in the red and blue light for $48 \mathrm{~h}$. A, Three-dimensional trajectory. B, Tending to blue light type. C, Tending to red light type. BL, blue light; RL, red light. $\cdot$, origin position of coordinates. Light source center went through the origin of coordinates and short dash arrows indicate the light source direction. Solid arrows indicate the direction of the movement.

素(Mccomb, 1962; Weiler et al., 1993; Atala \& Gianoli, 2008; Iida et al., 2018)等方面对植物的环旋运 动的机理进行了大量的研究。不同光质下环旋运动 方式的研究, 有助于加深对环旋运动意义的理解。

\section{1 光质对环旋运动方式的影响}

一般把植物的环旋运动描述为粗圆形、钟摆形 等(Brown et al., 1990), 多从二维平面上对运动进行 描述(Buda et al., 2003; Charzewska \& Zawadzki, 2006; Solheim et al., 2006; Stolarz \& Dziubińska, 2017)。近年来研究者注重在三维空间上描述环旋运 动(Gill et al., 2009)。本研究参考前人三维观测方法 自制了一个可连续观测, 不影响豇豆幼苗生长的装 置。通过观测发现, 豇豆幼苗在黑暗情况下有逆时 针螺旋上升运动和不规则的摆动上升运动两种形式 (1:2)。白光下为有规律的摆动上升运动, 摆幅先减 小后增大, 这种运动趋向光源。红光下, 豇豆幼苗运
动分解为近直线趋向光源运动和垂直光源方向摆动 上升运动。蓝光下主要为折线趋向光源上升运动。 红-蓝光下, 豇豆幼苗先趋向蓝光又转向红光方向 运动, 但向红光运动趋势不明显。

有研究显示向日葵椭圆形运动的摆幅变化在 1-8 cm (刘公社和张俊英, 1994), 本试验测得豇豆 幼苗最大摆幅为 $2.0 \mathrm{~cm}$, 最小摆幅为 $0.5 \mathrm{~cm}$ 。本试验 还测得白光下摆动运动的摆动幅度为 $0.1-0.6 \mathrm{~cm}$, 红光下垂直光源方向摆动的幅度为 $2.0-3.5 \mathrm{~cm}$ 。

因试验条件和植物种类的差异, 幼苗环旋一周 的时间是不同的。例如, 有人在地球上测得向日葵 幼苗环旋运动一周的时间为 $130 \mathrm{~min}$ (Israelsson \& Johnsson, 1967), 也有人测得环旋一周大多需要 180-240 min, 偶尔为80 min (刘公社和张俊英, 1994), 在太空中基本为 88 min (Brown et al., 1990)。 而本研究测得不同光质下, 豇豆幼苗环旋一周耗时 
最短的情况为黑暗情况下的螺旋上升运动, 环旋一 周为 $(82.7 \pm 4.2) \mathrm{min}$, 最长的是红光情况下的近直 线趋向光源运动, 环旋一周达 $(21.9 \pm 1.63) \mathrm{h}$ 。本试 验进一步表明, 不同光质下豇豆幼苗增长高度是不 同的，在 $48 \mathrm{~h}$ 内由高到低的顺序为: 红光 $>$ 黑暗>蓝 光>白光 $>$ 红-蓝光, 红蓝光组合有利于豇豆形态的 建成。

不仅不同种植物幼苗环旋运动的摆幅是有差异 的, 而且光质对植物幼苗环旋运动的形式和摆幅都 有影响, 相同植物个体间也有差异。这些影响方式 的总结和归类, 以及生态效应等需要进一步观测分 析。

\section{2 光质引起豇豆幼苗环旋运动变化的意义}

Galston (1980)以踠豆幼苗为材料, 发现蓝光能 够提高踠豆须环旋运动的速率, 单向的蓝光会改变 植物器官两边的运动相对幅度, 红光也能改变其幅 度。踠豆须环旋运动有利于被遮光叶片改变角度获 取阳光(Jaffe, 1970)。这种运动的结果是通过调整相 邻器官位置实现获取资源的结果, 显示出植株在获 取资源上器官间是相互协调配合的。本研究发现豇 豆幼苗在黑暗条件下仍然存在典型的环旋上升运动 和无规律的摆动上升运动, 这表明豇豆幼苗的运动 是一种自发的行为, 无须光信号启动。任何运动都 要消耗能量, 在黑暗条件下豇豆幼苗在较大幅度环 旋运动 $18.3 \mathrm{~h}$ 后, 变为运动幅度较小的不规则摆动 上升运动。这种运动方式的转变, 是由于前期在较 大空间中运动没有接收到可获取光源信号, 而主动 调节运动方式, 寻求更多的机会, 还是由于种子咜 存物质转化为幼苗可利用能量下降, 而失去运动能 力, 值得进一步的分析。此外, 豇豆幼苗在黑暗条件 下的自发运动, 不仅有螺旋上升运动, 还存在不规 则的摆动上升运动(1:2), 这种个体间运动形式的差 异, 是由于种子营养物质量的差异形成的, 还是随 机出现的, 也需要进一步的研究。

白光、红光和蓝光均改变了豇豆幼苗在黑暗中 的自发运动形式, 都使环旋的幅度下降, 趋光源性 显著, 哣豆幼苗的环旋运动是受可见光源方向调节 的。经典理论认为, 蓝光对向光性运动最有效(杨世 杰, 2000), 本研究虽然证实了蓝光确实对豇豆幼苗 的向光性是有效的, 但与白光、红光差异不大。在红蓝光源反向试验中, 甚至红光对向光性的有效性比 蓝光略强。
经典的理论认为植物向光性运动是由于光诱导 引起生长素不均匀分布造成的(杨世杰, 2000), 豇豆 幼苗黑暗条件下维持着无明显方向性的环旋上升生 长运动, 这种自发性的运动是否也受生长激素的调 节? 或者说进一步分析是什么因素调节豇豆幼苗在 黑暗条件下的自发运动, 对进一步深入认识植物环 旋运动的机制和生物学意义有重要价值。

本研究采用固定光源位置, 光质和照度观测豇 豆幼苗生长运动, 而在自然条件下, 太阳的位置、光 质比例和照度是不断变化的。要想更好地探索植物 环旋运动的奥秘, 进一步设计移动式可调节光源的 观测方法, 使观测结果更接近实际, 是值得进行探 索的一个方向。

致谢 华南农业大学本科生杨艳、郭永乐参与了本 论文的试验观测, 在此表示感谢。

\section{参考文献}

Atala CA, Gianoli EG (2008). Induced twining in Convolvulaceae climbing plants in response to leaf. Botany, 86, 595-602.

Brown AH (1993). Circumnutations: From Darwin to space flight. Plant Physiology, 101, 345-348.

Brown AH, Chapman DK (1977). Effects of increased gravity force on nutations of sunflower hypocotyls. Plant Physiology, 59, 636-640.

Brown AH, Chapman DK, Lewis RF, Venditti AL (1990). Circumnutations of sunflower hypocotyls in satellite orbit. Plant Physiology, 94, 233-238.

Buda A, Zawadzki T, Krupa M, Stolarz M, Okulski W (2003). Daily and infradian rhythms of circumnutation intensity in Helianthus annuus. Physiologia Plantarum, 119, 582-589.

Charzewska A, Zawadzki T (2006). Circadian modulation of circumnutation length, period, and shape in Helianthus annuus. Journal of Plant Growth Regulation, 25, 324-331.

Darwin C (1865). On the movements and habits of climbing plants. Botanical Journal of the Linnean Society, 33-34, $1-118$.

Darwin C (1876). The Movements and Habits of Climbing Plants. D. Appleton, New York.

Darwin C, Darwin F (1880). The Power of Movement in Plants. John Murray, London.

Galston AW (1980). Circumnutation, rhythms and lightregulated movements in plants. In: Skoog F ed. Plant Growth Substances 1979. Proceedings in Life Sciences. Springer, Berlin, Heidelberg. 437-443.

Gill AS, Bengough G, Grinev D, Dupuy L (2009). Imaging the $3 \mathrm{D}$ kinematics of circumnutation in maize roots. Comparative Biochemistry and Physiology Part A: Molecular 
\& Integrative Physiology, 153(Suppl.), 225.

Iida M, Takano T, Matsuura T, Mori IC, Takagi S (2018). Circumnutation and distribution of phytohormones in $\mathrm{Vi}$ gna angularis epicotyls. Journal of Plant Research, 131, 165-178.

Israelsson D, Johnsson A (1967). A theory for circumnutations in Helianthus annuus. Physiologia Plantarum, 20, 957-976.

Jaffe MJ (1970). On heliotropism in tendrils of Pisum sativum: A response to infrared irradiation. Planta, 92, 146.

Johnsson A, Israelsson D (2010). Application of a theory for circumnutations to geotropic movements. Physiologia Plantarum, 21, 282-291.

Johnsson A, Solheim BGB, Iversen TH (2009). Gravity amplifies and microgravity decreases circumnutations in Arabidopsis thaliana stems: Results from a space experiment. New Phytologist, 182, 621-629.

Kim HJ, Kobayashi A, Fujii N, Miyazawa Y, Takahashi H (2016). Gravitropic response and circumnutation in pea (Pisum sativum) seedling roots. Physiologia Plantarum, 157, 108-118.

Liu GS, Zhang JY (1994). The three-dimension track of circumnutation in sunflower seedlings. Acta Botanica Sinica, 12, 918-923. [刘公社, 张俊英 (1994). 向日葵幼苗环旋 运动的三维轨迹. 植物学报, 12, 918-923.]

Mccomb AJ (1962). An effect of gibberellic acid on girgumnutation. New Phytologist, 61, 128-131.

Melin D (1975). Growth and revolutive movement of shoots from Periploca graeca L. Zeitschrift Für Pflanzenphysiologie, 76, 384-399.

Scherer GF, Pietrzyk P (2014). Gravity-dependent differentiation and root coils in Arabidopsis thaliana wild type and phospholipase-A-I knockdown mutant grown on the International Space Station. Plant Biology, 16, 97-106.

Skotheim JM, Mahadevan L (2005). Physical limits and design principles for plant and fungal movements. Science, 308, $1308-1310$.

Solheim BGB, Kittang AI, Iversen TH, Johnsson A (2006). Preparatory experiments of Arabidopsis circumnutations in microgravity. Acta Astronautica, 59, 46-53.

Stolarz M, Dziubińska H (2017). Spontaneous action potentials and circumnutation in Helianthus annuus. Acta Physiologiae Plantarum, 39, 234.

Weiler EW, Albrecht T, Groth B, Xia ZQ, Luxem M, Liß H, Andert L, Spengler P (1993). Evidence for the involvement of jasmonates and their octadecanoid precursors in the tendril coiling response of Bryonia dioica. Phytochemistry, 32, 591-600.

Yoshihara T, Iino M (2006). Circumnutation of rice coleoptiles: Its relationships with gravitropism and absence in lazy mutants. Plant, Cell \& Environment, 29, 778-792.

Yang SJ (2000). Plant Biology. Science Press, Beijing. [杨世杰 (2000). 植物生物学. 科学出版社, 北京.]

责任编委：曹坤芳 责任编辑: 李 敏 